\title{
Complementary and alternative medicine use by Japanese children with pediatric surgical diseases
}

\author{
Keiichi Uchida ${ }^{*}$, Mikihiro Inoue, Kohei Otake, Yuhki Koike, Masato Kusunoki \\ Department of Gastrointestinal and Pediatric Surgery, Mie University Graduate School of Medicine, Tsu, Japan \\ Email: ${ }^{*}$ ucchie@clin.medic.mie-u.ac.jp
}

Received 28 January 2013; revised 1 March 2013; accepted 10 March 2013

Copyright (c) 2013 Keiichi Uchida et al. This is an open access article distributed under the Creative Commons Attribution License, which permits unrestricted use, distribution, and reproduction in any medium, provided the original work is properly cited.

\begin{abstract}
Background: This study aimed to survey the use of complementary and alternative medicine (CAM) therapies in Japanese children with major pediatric surgical diseases. Methods: This survey was mailed to each family of 355 patients. Results: One hundred fifty-three $(43 \%)$ of 355 mailed questionnaires were completely returned. Forty-one (27\%) of 153 parents responded that they used CAM for their child. There was no significant difference in CAM use according to sex, mean age, follow-up duration, family CAM users, and associated disorders between CAM users and non-users. Children with inflammatory bowel disease (IBD) were significantly more likely to use CAM than children without IBD (65\% vs $21 \%$, p = $0.0001)$. Only $20 \%$ of parents informed the attending physicians about CAM before its use. Conclusions: It is important for physicians caring for children with pediatric surgical disease, especially IBD, to be familiar with common forms of CAM.
\end{abstract}

Keywords: Complementary and Alternative Medicine; Children; Pediatric Surgery

\section{INTRODUCTION}

Interest in the use of complementary and alternative medicine (CAM) has greatly increased in developed countries for the last 2 decades [1,2]. It is very difficult to define the term of CAM because it includes a broad spectrum of alternative medical systems, biologically based therapies, mind-body interventions, manipulative and body-based methods, and energy therapies. There are various types of CAM used by individuals according to the nationality and the locality.

Studies on the use of CAM in healthy children and

"Corresponding author. adolescents have been reported [3-6]. Recently, evidence of the use of CAM at home in pediatric patients with special health care or children visiting the emergency department is accumulating in North America [7-14], Europe [15-19], and Australia [20]. It has been reported that children with chronic disease are more than three times more likely to use CAM compared with children without chronic diseases [15].

This study aimed to evaluate the use of CAM therapies at home in Japanese children with major pediatric surgical diseases in a single center experience. We also investigated which types of CAM were used, the reason for their use, information sources about CAM, the efficacy for the children, and if parents informed the attending physicians about their use of CAM.

\section{PATIENTS AND METHODS}

\subsection{Description of Patient Group}

Between April 1990 and December 2010, we experienced 482 major pediatric surgical cases such as congenital digestive duct atresia, abdominal wall abnormality, biliary atresia and dilatation, pediatric malignant tumors, and inflammatory bowel diseases (IBD). Patients who were lost to follow-up, those whose addresses were unknown, and those who died were excluded in this study. A total of 355 patients, who received follow-up care at our outpatient clinic, were included in this study.

\subsection{Questionnaire}

This survey was mailed to each family with an explanatory covering letter, asking each parent to complete the questionnaire on behalf of the child. Instructions were included to aid in the completion of the questionnaire. Parents were provided with a stamped addressed envelope and asked to return the questionnaire anonymously to the investigators. In the questionnaire, basic background data included the child's age, sex, the specific 
diagnosis and experience of using CAM in other family members. To ensure anonymity, forms were not numbered and parents were asked not to add any identifying information to the surveys. The questionnaire was prefaced with a brief introduction to define the term of CAM and to inform that this was a survey with consent.

CAM included healthy food diet, supplements, herbal medicine, vitamins, gigong/reiki, thermotherapy, massage therapy, chiropractic, music therapy, acupuncture, relaxation, and others. Prescribed enteral nutrition formula, vitamins, herbal medicines were excluded because we did not judge to need for patients.

At first, parents were asked whether they had any experience in using CAM in the management of the child's healthy condition. If they answered "no", the survey was finished in such a case. If they answered "yes", they continued to answer the questions. A list of types of CAM was given on the next page and the parents were asked to select the types of CAM used. They were also asked why they had chosen to use CAM and how they had obtained information about CAM. They were then asked whether they expressed the desire to discuss CAM with the attending doctor at our institute. Finally, they were asked how effective they perceived CAM to be, and to select one of the four following options: 1) continuing the use of CAM because of apparent effectiveness; 2) continuing the use of CAM although the effectiveness is unclear; 3 ) stopping the use of CAM because of unclear effectiveness; and 4) stopping the use of CAM because of apparent ineffectiveness.

At the end of the questionnaire, parents were permitted to mention their concerns, opinions and impressions about CAM.

\subsection{Statistics}

The chi square test was used in comparison of observed differences between groups. Tests were considered statistically significant at $\mathrm{p}<0.05$.

\section{RESULTS}

\subsection{Patients' Characteristics}

One hundred fifty-five (44\%) of 355 mailed questionnaire were returned. The specific diagnosis was not written in two of 155 questionnaires. A total of 153 questionnaires were completed by parents and returned by mail to the investigators. The mean age of the responders was $9.9 \pm 5.8$ years (mean $\pm \mathrm{SD}$; range, 1 - 27 years). The disease diagnosis of children in this survey included ulcerative colitis in 12, Crohn's disease in five, esophageal atresia in 21, duodenal atresia/stricture in 11, intestinal atresia in 21, anal atresia in 27, biliary atresia in 20 , biliary dilatation in 13 , omphalocele in five, gas- troschisis in six, and a malignant solid tumor in 23. Ten patients had one or two associated surgical diseases.

\subsection{The Use of CAM}

Forty-one (27\%) of 153 parents responded that they had used CAM for their child. Table 1 shows the comparison between 41 CAM users and 112 CAM non-users. There was no significant difference in CAM use according to sex, mean age, follow-up duration of disease, family CAM users, and associated disease or anomaly between CAM users and non-users.

Table 2 shows the use of CAM in each pediatric surgical disease. In this series, patients with IBD had the highest rate of the use (65\%), especially Crohn's disease (80\%). In children with congenital digestive duct atresia

Table 1. The comparison between complementary and alternative medicine (CAM) users and non-users.

\begin{tabular}{cccc}
\hline Characteristics & $\begin{array}{c}\text { CAM users } \\
(\mathrm{n}=41)\end{array}$ & $\begin{array}{c}\text { CAM non-users } \\
(\mathrm{n}=112)\end{array}$ & p value \\
\hline Gende (M/F) & $\begin{array}{c}18 / 23 \\
(44 \% / 56 \%)\end{array}$ & $\begin{array}{c}47 / 65 \\
(42 \% / 58 \%)\end{array}$ & n.s. \\
Age (years, mean \pm SD) & $10.5 \pm 5.8$ & $9.7 \pm 5.7$ & n.s. \\
$\begin{array}{c}\text { Duration of disease } \\
\text { (years, median) }\end{array}$ & 5.0 & 6.0 & n.s. \\
$\begin{array}{c}\text { Family CAM users } \\
\text { Associated disorders }\end{array}$ & $8(19 \%)$ & $15(13 \%)$ & n.s. \\
\hline
\end{tabular}

Table 2. The use of complementary and alternative medicine (CAM) in each pediatric surgical disease.

\begin{tabular}{ccc}
\hline Diseases & Use rate $^{*}$ & $\begin{array}{c}\text { Number of agents } \\
\text { used }^{\#}\end{array}$ \\
\hline IBD & $65 \%(11 / 17)$ & \\
lcerative colitis & $58 \%(7 / 12)$ & $2.4(1-5)$ \\
Crohn's disease & $80 \%(4 / 5)$ & $4.0(1-6)$ \\
Non-IBD & $21 \%(26 / 124)$ & \\
Esophageal atresia & $24 \%(5 / 21)$ & $2.8(1-5)$ \\
Duodena; atresia/stenosis & $18 \%(2 / 11)$ & $2.5(2-3)$ \\
Intestinal atresia & $19 \%(4 / 21)$ & $2.3(1-3)$ \\
Anal atresia & $15 \%(4 / 27)$ & $2.5(1-4)$ \\
Biliary atresia & $20 \%(4 / 20)$ & $3.0(1-8)$ \\
Biliary dilatation & $23 \%(3 / 13)$ & $1.3(1-2)$ \\
Omphalocele & $20 \%(1 / 5)$ & $1.3(1-2)$ \\
Gastroschisis & $16 \%(1 / 6)$ & 1 \\
Malignant solid tumor & $17 \%(4 / 23)$ & \\
\hline
\end{tabular}

IBD; Inflammatory bowel disease. *Use rate were expressed by percentage (users/responders). "Number of agents used was expressed by mean (range). 
and biliary disorders, CAM was used in 15\% - 24\%. A total of $17 \%$ of patients used CAM for malignant solid tumors. These data demonstrated that children with IBD were significantly more likely to use CAM than children without IBD (65\% (11/17) vs 21\% (26/124), p = 0.0001).

Children who used CAM agents used a mean of $2.4 \pm$ 1.7 types of agents. For each disease, the number of CAM agents used was highest in children with Crohn's disease (mean, 4; range, 1 - 6), but this was not statistically significant compared with other diseases. The number of CAM agents used in patients was one agent in 17 patients (41\%), two agents in nine (22\%), three agents in six $(15 \%)$, four agents in three (7\%), six agents in three $(7 \%)$, and six or greater agents in three $(7 \%)$.

\subsection{CAM Therapies}

Table 3 shows the types of CAM reported by the responders. Thirty of 41 parents (70\%) used CAM for the purpose of providing a benefit for their children with pediatric surgical diseases. Other parents (30\%) used CAM for general healthy condition. The frequently identified CAMs were a health-food dietdiet (37\%), supplements (31\%), non-prescribed herbal medicine (16\%), vitamins (16\%), gigong/reiki (10\%), thermotherapy (10\%), and massage therapy (10\%). Responders also suggested other CAMs, such as chiropractics (7\%), music therapy (5\%), acupuncture (2\%), and relaxation (2\%). Eight of 41 patients received herbal medicine for their child prescribed by physicians.

\subsection{Information Sources of CAM}

Table 4 shows how parents had obtained the information

Table 3. Complementary and alternative medicine (CAM) reported by respondes.

\begin{tabular}{ccc}
\hline & Number of Responders (\%) \\
\hline Healthy-food Diet & 15 & $37 \%$ \\
Supplement & 13 & $31 \%$ \\
Herbal medicine (Not prescribed) & 7 & $16 \%$ \\
Vitamins & 7 & $16 \%$ \\
Gigong/Reiki & 4 & $10 \%$ \\
Thermotherapy & 4 & $10 \%$ \\
Massage therapy & 4 & $10 \%$ \\
Chiropractic & 3 & $7 \%$ \\
Music therapy & 2 & $5 \%$ \\
Acupuncture & 1 & $2 \%$ \\
Relaxation & 1 & $2 \%$ \\
\hline
\end{tabular}

Table 4. Information sources of complementary and alternative medicine (CAM) used.

\begin{tabular}{ccc}
\hline & \multicolumn{2}{c}{ Number of Responders (\%) } \\
\hline Acqaintances & 22 & $56 \%$ \\
Publications & 4 & $10 \%$ \\
Internet & 3 & $8 \%$ \\
Pharmacy & 3 & $8 \%$ \\
TV commercial & 2 & $5 \%$ \\
Others & 5 & $13 \%$ \\
Total & 39 & \\
\hline
\end{tabular}

about CAM. Parents most commonly obtained information about CAM from acquaintances (56\%). Other sources included publications (10\%), the Internet (8\%), the pharmacy (8\%), TV commercials (5\%), and others (13\%).

Twenty-five of 41 parents (61\%) did not express the desire to discuss CAM with the attending physician at our institute before the use of CAM. Eight of 41 parents (20\%) informed the attending physicians about CAM before their use.

\subsection{Efficacy of CAM}

Twelve parents (25\%) answered that they are continuing the use of CAM because of the apparent effectiveness at the time of the survey. Nineteen parents (39\%) answered that they are continuing to use CAM, although the effectiveness was unclear at the time of the survey. Thirteen parents (27\%) answered that they stopped the use of CAM because of unclear effectiveness.

Four parents (9\%) answered that they stopped the use of CAM because adverse events appeared or they felt that the CAM used was not suitable for their child. Two parents who used herbal medicine reported adverse effects such as diarrhea and eruption.

\subsection{Concerns and Opinions of CAM}

In this survey, there were no questions about the specific costs of therapies. However, one responder commented about the unclear effectiveness and the high cost of CAM for their child. Three parents were more willing to consider using herbal medicine if the symptoms of the disease in their children were more severe in the future.

\section{DISCUSSION}

Pediatric surgical diseases sometimes become chronic and refractory after an operation and become problematic in children for a long time. The present study demonstrated that CAM use in Japanese patients who had un- 
dergone operation for pediatric surgical disease had no relationship with sex, age, duration of disease, parents' use, and associated disorders. It has been previously reported that factors associated with pediatric use of CAM are a higher age $[4,6]$, the patient's self-reported overall health [10], white ethnicity [10], higher education in the parent's career $[4,6,10,14]$, use of prescribed medication [4], and use of CAM by a parent $[4,6,15]$.

The prevalence of CAM in children is variable. Several large studies in the pediatric population have demonstrated that CAM is used for symptom management, and healthmaintenance or prevention, rather than treatment of specificconditions [3,4,6,21]. Moreover, many studies have found a higher CAM use among children with chronic conditions such as cerebral palsy $[11,15]$, asthma [22], cancer [15,17,23,24], sicklecell anemia [25], IBD $[10,15,16,18,20]$, and emergent illness $[14,21]$. The present study demonstrated that $27 \%$ of pediatric surgical patients had an experience of using CAM and the rate of CAM use in IBD patients was significantly higher compared with that in non-IBD patients. We consider that this is important data because there are no other studies that have compared the use of CAM between pediatric IBD and other disorders.

In the current study, children used a mean of 2.4 types of CAM. Three patients (7\%) used six or more types of CAM. Cotton et al. reported [7] that adolescents with more severe diseases and worse health-related quality of life might be more willing to consider mind-body CAM for future symptom management. We recognize a limitation of this study. Death cases were not included in the subjects. Parents in death cases might use CAM more aggressively compared with parents whose child is living.

Popular CAM modalities used in this study were a health-food diet, supplements, herbal medicine, and vitamins, which is consistent with other studies $[10,16,17]$. However, spiritual interventions such as religious help and prayer have been reported in several studies $[7,10$, 11,14,17]. Many parents used CAM for the purpose of providing a benefit or hoping for a cure in this study. Other common reasons for CAM use were reported side effects from prescribed medicines not working as well as they had hoped [16,17,19,20]

To our surprise, many parents obtained information about CAM from their acquaintances in an informationoriented society and Internet era. Additionally, many CAM users did not express the desire to discuss CAM with the attending physician, and only $20 \%$ of parents informed the attending physicians about CAM before use in our study. In previous studies, $45 \%-83 \%$ of parents reported informing their child's physician of their use of CAM therapies $[13,14,19]$. In another previous study [26], 53\% of parents expressed the desire to discuss
CAM with their pediatrician, and this increased to $75 \%$ among those who used CAM themselves. It was reported that factors associated with increased disclosure to the pediatrician were CAM use in children younger than 6 years, bioenergetic CAM use, and parental CAM non-use [26-29]. It is important that physicians should have more communication with parents about CAM use for pediatric patients.

The most important study to conduct in pediatric patients who use CAM is clinical outcome research. However, it is very difficult to evaluate the efficacy of CAM because of the broad health outcome including morbidity, mortality, cost of care, patient satisfaction, cultural identify, spiritual beliefs, coping, and self-efficacy. The present study evaluated the self-efficacy of CAM, and 25\% of parents felt that CAM use was effective for their children, which is higher than that reported in another study [20]. In addition, four parents (9\%) answered that they stopped the use of CAM because adverse events appeared or they felt that the CAM used was not suitable for their child. Two parents who used herbal medicine reported adverse effects such as diarrhea and eruption. This questionnaire did not include questions about the specific costs of CAM therapies. However, one CAM user raised the issue of unclear effectiveness and the high cost of CAM for their child.

In conclusion, it is important for physicians caring for patients with pediatric surgical disease, especially IBD, to be familiar with common forms of CAM and to be able to provide general counseling to their parents about CAM use.

\section{REFERENCES}

[1] Eisenberg, D.M., Kessler, R.C., Foster, C., et al. (1993) Unconventional medicine in the United States. Prevalence, costs, and patterns of use. The New England Journal of Medicine, 328, 246-252. doi:10.1056/NEJM199301283280406

[2] Eisenberg, D.M., Davis, R.B., Ettner, S.L., et al. (1998) Trends in alternative medicine use in the United States, 1990-1997: Results of a follow-up national survey. JAMA, 280, 1569-1575. doi:10.1001/jama.280.18.1569

[3] Wilson, K.M., Klein, J.D., Sesselberg, T.S., et al. (2006) Use of complementary medicine and dietary supplements among U.S. adolescents. Journal of Adolescent Health, 38, 385-394. doi:10.1016/j.jadohealth.2005.01.010

[4] Spigelblatt, L., Laîné-Ammara, G., Pless, I.B., et al. (1994) The use of alternative medicine by children. Pediatrics, 94, 811-814.

[5] Kemper, K.J., Cassileth, B. and Ferris, T. (1999) Holistic pediatrics: A research agenda. Pediatrics, 103, 902-909.

[6] Birdee, G.S., Phillips, R.S., Davis, R.B., et al. (2010) Factors associated with pediatric use of complementary and alternative medicine. Pediatrics, 125, 249-256. 


\section{doi:10.1542/peds.2009-1406}

[7] Coton, S., Humenay Roberts, Y., Tsevat, J., et al. (2010) Mind-body complementary alternative medicine use and quality of life in adolescents with inflammatory bowel disease. Inflammatory Bowel Diseases, 16, 501-506.

[8] Hilsden, R.J., Verhoef, M.J., Rasmussen, H., et al. (2011) Use of complementary and alternative medicine by patients with inflammatory bowel disease. Inflammatory Bowel Diseases, 17, 655-662. doi:10.1002/ibd.21360

[9] Sencer, S.F. and Kelly, K.M. (2007) Complementary and alternative therapies in pediatric oncology. Pediatric Clinics of North America, 54, 1043-1060. doi:10.1016/j.pcl.2007.10.007

[10] Wong, A.P., Clark, A.L., Garnett, E.A., et al. (2009) Use of complementary medicine in pediatric patients with inflammatory bowel disease: Results from a multicenter survey. Journal of Pediatric Gastroenterology and Nutrition, 48, 55-60. doi:10.1097/MPG.0b013e318169330f

[11] Sanders, H., Davis, M.F., Duncan, B., et al. (2003) Use of complementary and alternative medical therapies among children with special health care needs in southern Arizona. Pediatrics, 111, 584-587. doi:10.1542/peds.111.3.584

[12] Moher, D., Soeken, K., Sampson, M., et al. (2002) Assessing the quality of reports of systematic reviews in pediatric complementary and alternative medicine. BMC Pediatrics, 2, 3. doi:10.1186/1471-2431-2-3

[13] Pitetti, R., Singh, S., Hornyak, D., et al. (2001) Complementary and alternative medicine use in children. Pediatric Emergency Care, 17, 165-169. doi:10.1097/00006565-200106000-00004

[14] Losier, A., Taylor, B. and Fernandez, C.V. (2005) Use of alternative therapies by patients presenting to a pediatric emergency department. The Journal of Emergency Medicine, 28, 267-271. doi:10.1016/j.jemermed.2004.11.019

[15] McCann, L.J. and Newell, S.J. (2006) Survey of paediatric complementary and alternative medicine use in health and chronic illness. Archives of Disease in Childhood, 91, 173-174. doi:10.1136/adc.2004.052514

[16] Heuschkel, R., Afzal, N., Wuerth, A., et al. (2002) Complementary medicine use in children and young adults with inflammatory bowel disease. The American Journal of Gastroenterology, 97, 382-388. doi:10.1111/j.1572-0241.2002.05474.X

[17] Bishop, F.L., Prescott, P., Chan, Y.K., et al. (2010) Prevalence of complementary medicine use in pediatric cancer: A systematic review. Pediatrics, 125, 768-776. doi:10.1542/peds.2009-1775

[18] Joos, S., Rosemann, T., Szecsenyi, J., et al. (2006) Use of complementary and alternative medicine in Germany: A survey of patients with inflammatory bowel disease. BMC Complementary and Alternative Medicine, 6, 19. doi:10.1186/1472-6882-6-19

[19] Cincotta, D.R., Crawford, N.W., Lim, A., et al. (2006) Comparison of complementary and alternative medicine use: Reasons and motivations between two tertiary children's hospitals. Archives of Disease in Childhood, 91, 153-158. doi:10.1136/adc.2005.074872

[20] Day, A.S., Whitten, K.E. and Bohane, T.D. (2004) Use of complementary and alternative medicines by children and adolescents with inflammatory bowel disease. Journal of Paediatrics and Child Health, 40, 681-684. doi:10.1111/j.1440-1754.2004.00510.x

[21] Barnes, P.M., Bloom, B. and Nahin, R.L. (2007) Complementary and alternative medicine use among adults and children: United States, 2007. National Health Statistics Reports, 12, 1-23.

[22] Braganza, S., Ozuah, P.O. and Sharif, I. (2003) The use of complementary therapies in inner-city asthmatic children. Journal of Asthma, 40, 823-827. doi:10.1081/JAS-120023574

[23] Hamidah, A., Rustam, Z.A., Tamil, A.M., et al. (2009) Prevalence and parental perceptions of complementary and alternative medicine use by children with cancer in a multi-ethnic Southeast Asian population. Pediatric Blood \& Cancer, 52, 70-74. doi:10.1002/pbc. 21798

[24] Laengler, A., Spix, C., Seifert, G., et al. (2008) Complementary and alternative treatment methods in children with cancer: A population-based retrospective survey on the prevalence of use in Germany. European Journal of Cancer, 44, 2233-2240. doi:10.1016/j.ejca.2008.07.020

[25] Sibinga, E.M., Shindell, D.L., Casella, J.F., et al. (2006) Pediatric patients with sickle cell disease: Use of complementary and alternative therapies. Journal of Alternative and Complementary Medicine, 12, 291-298. doi:10.1089/acm.2006.12.291

[26] Sibinga, E.M., Ottolini, M.C., Duggan, A.K., et al. (2004) Parent-pediatrician communication about complementary and alternative medicine use for children. Clinical Pediatrics, 43, 367-373. doi:10.1177/000992280404300408

[27] Dokken, D. and Sydnor-Greenberg, N. (2000) Exploring complementary and alternative medicine in pediatrics: Parents and professionals working together for new understanding. Pediatric Nursing Journal, 26, 383-390.

[28] Committee on Children with Disabilities (2010) American Academy of Pediatrics: Counseling families who choose complementary and alternative medicine for their child with chronic illness or disability. Committee on Children with Disabilities. Pediatrics, 107, 598-601.

[29] O’Keefe, M. and Coat, S. (2010) Increasing health-care options: The perspectives of parents who use complementary and alternative medicines. Journal of Paediatrics and Child Health, 46, 296-300. doi:10.1111/j.1440-1754.2010.01711.x 